\title{
Acesso realizado ao Programa de Saúde da Família em área com "alta" cobertura do subsistema privado
}

\author{
Access to the Family Healthcare Program in an area \\ with "extensive" coverage of the private healthcare system
}

Aylene Bousquat ${ }^{1}$

Adriana Gomes ${ }^{1}$

Maria Cecilia Goi Porto Alves ${ }^{2}$

${ }^{1}$ Programa de Pós-

Graduação em Saúde

Coletiva, Universidade

Católica de Santos. R.

Airosa Galvão 64, Água

Branca. 05002-070 São

Paulo SP.aylene.bousquat@

pesquisador.cnpq.br

${ }^{2}$ Instituto de Saúde,

Secretaria de Estado da

Saúde de São Paulo.

\begin{abstract}
The access profile to the various services offered by the Family Health Program (PSF) in an area with extensive private health system coverage is studied in the city of Santo André, state of São Paulo. The sample was studied in two phases based on the assumption that the use of the activities offered by the Family Health Program is not uniform. Residual, full and partial population groups were therefore considered. Interviews were conducted with 175 individuals and the registered population is composed of full (0.5\%), partial (31.0\%) and residual (68.5\%) users. In the bivariate analysis, significant differences were observed between partial and residual groups in relation to age, income, employment, affiliation to the private subsystem and years of schooling. However, the ratios of prevalence when estimated by the Poisson multiple model indicate that the factors that influence the access profile of the PSF activities are private subsystem membership and income. This highlights the fact that some services offered do not reach more than $20 \%$ of the population. Despite the immense potential of innovative proposals incorporated by the PSF, this study shows that there is selective use of its activities by the population, strongly influenced by private subsystem affiliation and income.
\end{abstract}

Key words Family Health Program, Access to health services, Primary healthcare
Resumo O perfil do acesso realizado aos distintos serviços ofertados pelo PSF em área com importante cobertura do sistema privado é identificado em unidade de saúde na cidade de Santo André, estado de São Paulo. A amostra, em duas fases, foi delineada partindo do pressuposto de que o uso possivel das atividades ofertadas pela estratégia saúde da família não é uniforme, com base neste uso foram considerados três grupos populacionais: residual, parcial e completo. Foram entrevistadas 175 pessoas, a população cadastrada é composta por 0,5\% de usuários com perfil completo, 31,0\% parcial e $68,5 \%$ residual. Na análise bivariada observaram-se diferenças significantes entre os grupos parcial e residual em relação à idade, renda, trabalho, afiliação ao subsistema privado e anos de escolaridade. No entanto, as razões de prevalência, quando estimadas por meio do modelo múltiplo de Poisson indicam que os fatores que influem no perfil de acesso são afiliação ao subsistema privado e renda. Chama atenção que alguns serviços ofertados não alcancem mais de $20 \%$ da população cadastrada. Apesar da imensa potencialidade das propostas inovadoras incorporadas pelo PSF, observa-se uso seletivo de suas atividades pela população, fortemente influenciado pela afiliação ao subsistema privado e pela renda.

Palavras-chave Programa de saúde da família, Serviços de saúde, Acesso aos serviços de saúde, Atenção primária à saúde 


\section{Introdução}

O Programa de Saúde da Família (PSF), principal estratégia de estruturação da atenção básica no Brasil, vem sendo objeto de diversos estudos desde sua implantação em 1994. Os enfoques utilizados foram e são muito diversos; entre outros citam-se as potencialidades do programa em promover mudanças no modelo da atenção básica ${ }^{1}$; características de seu financiamento ${ }^{2}$; avaliação de seu desempenho ${ }^{3}$; seu status como politica ${ }^{4,5}$; impacto no perfil de morbidade e mortalidade da populaçã $0^{6,7}$; constrangimentos e potencialidades de sua implantação em áreas urbanas ${ }^{8,9}$.

No entanto, dois aspectos importantes ainda não foram suficientemente explorados. O primeiro reside no detalhamento do acesso da população cadastrada às diferentes atividades de atenção à saúde propostas pela Estratégia da Saúde da Família, que vão além das consultas médicas. O segundo é o entendimento de como o acesso a este rol ampliado de atividades ocorre em áreas nas quais parcela da população cadastrada é afiliada ao Subsistema Privado da Saúde.

É exatamente a proposta de diversidade de atividades e o trabalho multiprofissional, rompendo os muros das unidades de saúde, que tornam o PSF uma experiência inovadora no cenário internacional da $\mathrm{APS}^{10,11}$. Esta característica virtuosa merece ser incorporada aos estudos sobre acesso e utilização dos serviços da ESF. Utilizando-se a terminologia proposta por Andersen ${ }^{12}$, quais são os diferentes perfis do acesso realizado? Toda a população cadastrada acessa este conjunto de iniciativas? Quais são as possíveis explicações para acessos realizados distintos? Concordamos com Fernandes et al. ${ }^{13}$ quando afirmam que, no geral, as pesquisas sobre utilização de serviços de saúde priorizam a demanda presente nos serviços, excluindo pessoas que não os procuram, dificultando o real conhecimento do cenário. A opção por estudos com foco na população usuária das unidades de saúde assume o pressuposto, para o qual não há evidência empírica, de que ao se ofertar um serviço ele é utilizado por toda população cadastrada. Ademais, no caso do PSF, por sua proposta de atenção ampliada, é fundamental reconhecer o que acontece com a população cadastrada como um todo.

A partir do leque de atividades previstas para realização na ESF é possível considerar a existência de três possíveis padrões de acesso: completo, parcial e residual ${ }^{14}$. O primeiro é formado pela parcela da população cadastrada que frequenta a USF e realiza atividades de grupo e comunitári- as, além de receber a visita do Agente Comunitário de Saúde (ACS). O padrão parcial, além de receber a visita do ACS, frequenta a USF, notadamente utilizando consultas médicas individuais, porém não realiza atividades comunitárias, nem participa de atividades de grupos. Por último o padrão residual composto pela população cadastrada que não frequenta a USF, não realiza atividades comunitárias e não participa de atividades de grupos; recebendo a visita do Agente Comunitário de Saúde (ACS).

O segundo aspecto - do acesso a este rol ampliado de atividades pela população afiliada ao subsistema privado - é relevante, pois dialoga com o mix publico privado existente no sistema de saúde brasileiro. Santos et al. ${ }^{15}$ observam que a Constituição Federal de 1988 foi fortemente inspirada nos sistemas nacionais de saúde de acesso universal e integral; no entanto a existência de setor privado importante, tanto no campo da prestação de serviços, como no que tange ao asseguramento privado levou ao formato peculiar do sistema de saúde brasileiro, fundado como sistema nacional de saúde que, entretanto, mantém participação do setor público e do privado. E é com esta realidade que as ESF se confrontam em grande parte do território nacional. A fase inicial da implantação do PSF voltada aos municípios com Îndices de Desenvolvimento Humano baixos já foi superada há bastante tempo e o desafio da proposta reside na sua implantação nas grandes áreas urbanas, nas metrópoles brasileiras, nas quais a penetração do subsistema privado é bastante importante.

Diversos estudos conduzidos internacionalmente evidenciam diferenças na utilização de serviços de APS a depender da afiliação do entrevistado ao setor privado ${ }^{16-23}$. Nesta literatura destacamos os estudos espanhóis, pois tem como objeto de estudo um sistema de saúde nacional instituído na mesma época que o brasileiro e que também convive, embora em menor proporção, com a existência de um setor privado que pode ser considerado suplementar e duplicado ${ }^{24}$. Recorda-se que o Sistema Nacional de Saúde Espanhol foi criado em 1986 com caráter universal; as responsabilidades são divididas entre o Governo Central e as 17 Comunidades Autônomas. Tal como o Brasil, estas Comunidades tem populações e inserções econômicas bastante diferentes, sendo que no caso espanhol há ainda a coexistência de sentimentos nacionais distintos. Desde 1992 vem sendo executada uma grande reforma com a ampliação da APS. A cobertura do Sistema Nacional de Saúde é universal e cerca de 10,3\% 
da população possui também o chamado "aseguramiento libre" que agrupa entidades mercantis e mutualistas; no entanto em áreas economicamente mais dinâmicas, como a Catalunha, este percentual é bem superior.

Vários estudos brasileiros também demonstram diferenças no acesso aos serviços de saúde ambulatoriais, com percentuais superiores de usuários do subsistema privado comparado com os do SUS ${ }^{25-30}$. No presente artigo pretendemos aprofundar esta discussão, tomando por referência os diversos serviços oferecidos pelo PSF à população, não restringindo a análise apenas às consultas médicas. O objetivo é caracterizar o perfil do acesso realizado aos distintos serviços ofertados pelo PSF em área com importante cobertura do subsistema privado.

Os resultados aqui apresentados fazem parte da pesquisa "Avaliação do PSF pela população residente em áreas metropolitanas através de instrumento de coleta de dados informatizado" financiada pelo CNPq e que foi realizada nos municípios de São Paulo, Santo André e Praia Grande, todos no estado de São Paulo.

\section{Metodologia}

\section{Local do Estudo}

Para cumprir o objetivo proposto era fundamental que o local escolhido para a realização da pesquisa fosse um município com "alta" cobertura do subsistema privado, ou seja, cobertura bastante superior à média nacional. Dessa forma, foi selecionado o município de Santo André, localizado no grande $\mathrm{ABC}$ paulista, na região metropolitana de São Paulo (RMSP). Conta com uma população de 676.407 habitantes em $2010^{31}$, destes, aproximadamente $64,1 \%$ são afiliados ao subsistema privado, percentual superior tanto ao nacional quanto ao do estado de São Paulo.

A economia do município está centrada no setor de serviços, em seguida, na indústria, sendo que o setor agropecuário é residual. O Índice de Desenvolvimento Humano Municipal (IDHM) de Santo André, referente ao ano 2000, foi de 0,835 . A cidade possui o total de 299 estabelecimentos de saúde, dos quais 52 pertencentes à rede pública e 247 à rede privada. Quando da realização da pesquisa existiam dez unidades de saúde com PSF implantado.

\section{Escolha da Unidade}

Após a definição do município procedeu-se a escolha da USF a ser pesquisada. A definição desta foi feita através de processo de tipificação, ou seja, escolheu-se a unidade que mais se aproximava de uma característica do município de Santo André. Foram sistematizadas, para todas as unidades com PSF no município, as seguintes informações: origem da unidade (municipalizada ou não); modalidade de assistência (PSF pura ou PSF mista); existência de parceiro na gestão; idade da unidade e tempo de implantação do PSF; existência de atendimento após as 17 horas; formação do gerente da unidade; tempo de permanência do gerente no cargo; número de equipes PSF; número de equipes PACS; número de dentistas do PSF; existência de especialidade básica (clínico, pediatra e ginecologista); disponibilidade de equipe de saúde mental; situação física da unidade (boa, regular ou ruim); existência de rampa e banheiro para portadores de necessidades especiais; Conselho de Saúde em atividade; localização em área rural ou urbana. Estas informações foram fornecidas pela Secretaria Municipal de Saúde de Santo André.

A partir da análise destes dados foi definido o perfil da unidade-tipo que deveria aglutinar o maior número possível das seguintes características: unidade PSF exclusivo; gerente com formação profissional de médico ou enfermeiro; no máximo duas equipes PSF; sem Conselho de Saúde; localizada em área urbana; funcionar há mais de dois anos e menos de cinco anos. Foi escolhida a Unidade $\mathrm{X}$ e nesta a equipe de saúde da família definida foi a que atuava há mais tempo.

\section{Instrumento}

O instrumento aplicado foi elaborado para avaliar tanto o perfil de utilização dos serviços quanto a percepção da população sobre estes. Como uma das grandes inovações do PSF consiste em ofertar serviços não restritos à consulta médica individual - uma vez que busca fugir da abordagem queixa-conduta e propiciar atividades de promoção e prevenção à saúde - buscouse incorporar essa diversidade.

\section{Delineamento da amostra}

A amostra foi delineada partindo do pressuposto de que o uso que se faz de uma USF não é uniforme, pois na medida em que a unidade oferece serviços diferenciados, a população também 
se utiliza dos serviços de forma diferenciada, de acordo com suas necessidades e particularidades.

Dessa forma, foram considerados três grupos populacionais com base no tipo de uso residual, parcial e completo - descritos anteriormente.

Para a obtenção de amostras para esses grupos, foi utilizada amostragem em duas fases. Nesse processo de amostragem, sorteia-se uma primeira amostra da população de estudo, obtêm-se dados para a construção dos estratos de interesse e nestes, novas amostras são sorteadas.

$\mathrm{Na}$ primeira fase do estudo, foram sorteadas 819 pessoas a partir do cadastro do Sistema de Informação da Atenção Básica (SIAB), no qual havia o registro de 3274 moradores. Por meio de consultas aos ACS, os sorteados dessa primeira amostra, foram classificados em dois estratos. No primeiro, foram alocadas as pessoas que os ACS consideravam pertencentes ao grupo "uso completo" (7 pessoas) e no segundo, aquelas que, na opinião dos ACS, não fariam parte desse grupo (812 pessoas). $\mathrm{Na}$ fase seguinte do processo de amostragem, foram sorteadas 213 pessoas do segundo estrato e foram selecionadas todas as pertencentes ao primeiro estrato. As frações de amostragem foram as seguintes:

Primeiro estrato $\rightarrow$

$$
f_{1}=\frac{819}{3274} \cdot \frac{7}{7}=\frac{1}{3,9976}
$$

Segundo estrato $\rightarrow$

$$
f_{1}=\frac{819}{3274} \cdot \frac{213}{812}=\frac{1}{15,2395}
$$

As entrevistas, feitas nos domicílios nos quais residiam os sorteados, foram conduzidas face a face por entrevistadores treinados, que utilizaram suporte eletrônico para captura de dados in loco (palm-top). A partir das informações fornecidas pelos próprios entrevistados foi possível classificá-los nos grupos "uso completo", "uso parcial" e "uso restrito". No entanto, o número de usuários completos encontrado foi ínfimo (2), desta forma optou-se por não apresentar este grupo separadamente, tendo sido anexados ao grupo parcial.

$\mathrm{Na}$ análise dos dados, para compensar as diferentes probabilidades de seleção utilizadas nos dois estratos, foram introduzidos pesos, determinados pelo inverso das frações de amostragem.

Foi verificada, por meio do qui-quadrado, a existência de associação entre o tipo de uso e as variáveis independentes: idade, sexo, situação de trabalho, afiliação no subsistema privado, cor autorreferida, estado civil, número de moradores no domicílio, anos de escolaridade, autopercepção de saúde e faixa etária por meio do teste qui-quadrado. Razões de prevalência brutas e ajustadas foram estimadas utilizando o modelo de regressão de Poisson. Foram estimadas as prevalências do uso de serviço residual segundo as variáveis acima e as respectivas razões de prevalência, as estimativas das razões de prevalência brutas e ajustadas. Recorda-se que Barros e Hirakata $^{32}$ indicam que nos estudos transversais a regressão de Poisson gera medidas de mais fácil de interpretação pelos não especialistas; no caso de estudos no campo das políticas de saúde consideramos esta característica fundamental, para que os resultados possam ser apreendidos também pelos formuladores de políticas.

Foram identificadas também as atividades que a população entrevistada utilizou no último ano na Unidade da Saúde da Família, bem como os motivos de não utilização, além do uso de outros serviços de saúde no mesmo período Os entrevistados também foram questionados sobre suas percepções acerca das consultas médicas e das visitas do ACS.

Os dados foram analisados por meio do software Stata 11.0, levando em conta a ponderação dos dados.

\section{Resultados}

Foram entrevistadas 175 pessoas, sendo 2 usuários do grupo completo, 54 do parcial e 119 do residual. Considerando as frações de amostragem, a composição da população cadastrada é de $0,5 \%$ de usuários com perfil completo, $31,0 \%$ com perfil parcial e $68,5 \%$ do residual.

$\mathrm{Na}$ análise bivariada observaram-se diferenças estatisticamente significantes entre os grupos parcial e residual em relação à idade, renda, situação de trabalho e afiliação ao subsistema privado e anos de escolaridade. A diferença na composição por sexo entre os dois grupos fica no limite da significância com $\mathrm{p}=0,06$. (Tabela 1 ). As estimativas do uso residual do PSF indicam que esse uso é menor entre os que não trabalham, não possuem plano de saúde e tem renda inferior a 2 salários mínimos. Estes resultados são confirmados quando se pergunta aos moradores qual o motivo de não utilização da USF, a principal resposta é a afiliação ao subsistema privado $(61,6 \%)$ seguida de não necessidade de frequentar serviços de saúde devido a boa condição de saúde (13,3\%). 
Tabela 1. Distribuição percentual dos entrevistados segundo tipo de uso do PSF e variáveis demográficas, socioeconômicas e de saúde.

\begin{tabular}{|c|c|c|c|c|c|c|c|c|}
\hline \multirow[b]{2}{*}{ Variável } & \multirow[b]{2}{*}{ Categoria } & \multicolumn{2}{|c|}{ residual } & \multicolumn{2}{|c|}{ parcial completo } & \multicolumn{2}{|c|}{ parcial completo } & \multirow{2}{*}{$\begin{array}{c}\text { valor } \\
\text { de p }\end{array}$} \\
\hline & & $\mathbf{p}$ & $\mathrm{IC}_{95 \%}$ & $\mathbf{p}$ & $\mathrm{IC}_{95 \%}$ & $\mathbf{p}$ & IC $_{95 \%}$ & \\
\hline \multirow[t]{2}{*}{ Sexo } & feminino & 48.4 & $39.3-57.6$ & 64.7 & $49.6-77.3$ & 52.7 & $44.8-60.4$ & 0,066 \\
\hline & masculino & 51.6 & $42.4-60.7$ & 35.3 & $22.6-50.3$ & 47.3 & $39.6-55.1$ & \\
\hline \multirow[t]{4}{*}{ Idade } & $<20$ & 14.8 & $9.3-22.6$ & 26.4 & $15.4-41.6$ & 17.8 & $12.6-24.6$ & 0,005 \\
\hline & $20-39$ & 30.7 & $22.9-39.8$ & 21.3 & $11.5-35.9$ & 28.3 & $21.7-35.9$ & \\
\hline & $40-59$ & 39.2 & $30.6-48.5$ & 18.1 & $9.2-32.5$ & 33.7 & $26.7-41.6$ & \\
\hline & $60 e+$ & 15.3 & $9.8-23.1$ & 34.1 & $21.5-49.6$ & 20.2 & $14.6-27.2$ & \\
\hline \multirow[t]{4}{*}{ Situação conjugal } & casado & 44.9 & $34.9-54.1$ & 49.6 & $35.1-64.1$ & 46.1 & $38.4-53.9$ & 0.839 \\
\hline & separado & 8.9 & $4.9-15.9$ & 5.0 & $1.2-18.1$ & 7.9 & $4.5-13.5$ & \\
\hline & solteiro & 40.8 & $32.1-50.1$ & 39.1 & $25.9-54.1$ & 40.3 & $32.9-48.3$ & \\
\hline & viúvo & 5.4 & 2.4-11.5 & 6.3 & $2.0-18.1$ & 5.6 & $2.9-10.5$ & \\
\hline \multirow[t]{2}{*}{ Cor autorreferida } & branco & 85.8 & $78.0-91.2$ & 76.7 & $62.0-86.9$ & 83.4 & $76.7-88.5$ & 0.170 \\
\hline & pardo ou negro & 14.2 & $8.8-22.0$ & 23.3 & $13.1-38.0$ & 16.6 & $11.5-23.3$ & \\
\hline \multirow{2}{*}{ Trabalho } & não & 40.1 & $31.5-49.5$ & 68.5 & $53.4-80.6$ & 47.6 & $39.9-55.5$ & 0.002 \\
\hline & $\operatorname{sim}$ & 59.8 & $50.5-68.5$ & 31.4 & $19.4-46.6$ & 52.4 & $44.5-60.1$ & \\
\hline \multirow[t]{2}{*}{ Setor supletivo } & não & 18.2 & $12.2-26.3$ & 60.3 & $45.3-75.5$ & 29.3 & $22.7-36.9$ & $<0,001$ \\
\hline & $\operatorname{sim}$ & 81.8 & $73.7-87.8$ & 39.7 & $26.5-54.7$ & 70.7 & $63.1-77,3$ & \\
\hline \multirow[t]{5}{*}{ Renda } & menor 3 SM & 11.0 & $6.4-18.3$ & 8.4 & $34.1-63.0$ & 20.8 & $15.2-27.9$ & $<0,001$ \\
\hline & 3 a $5 \mathrm{SM}$ & 21.3 & $14.7-29.8$ & 27.6 & $16.3-42.8$ & 23.0 & $17.0-30.2$ & \\
\hline & 5 a $10 \mathrm{SM}$ & 41.0 & $32.3-50.3$ & 20.1 & $10.7-34.6$ & 35.5 & $28.4-43.4$ & \\
\hline & $10 \mathrm{ou}+\mathrm{SM}$ & 11.2 & $6.6-18.5$ & 1.3 & $0.3-5.3$ & 8.6 & $5.1-14.1$ & \\
\hline & não informada & 15.5 & $9.8-23.4$ & 2.5 & $0.3-16.0$ & 12.0 & $7.7-18.3$ & \\
\hline \multirow{4}{*}{$\begin{array}{l}\text { Escolaridade } \\
\text { (anos de estudo) }\end{array}$} & 0 a 7 & 27.3 & $19.9-36.3$ & 48.3 & $33.9-62.9$ & 32.9 & $25.9-40.6$ & 0,032 \\
\hline & 8 a 10 & 11.9 & $7.1-19.1$ & 12.1 & $5.7-24.0$ & 12.0 & $7.9-17.8$ & \\
\hline & 11 ou mais & 59.8 & $50.5-68.5$ & 36.4 & $23.5-51.6$ & 53.7 & $45.8-61.4$ & \\
\hline & não informado & 0.9 & $0.1-6.2$ & 3.2 & $0.6-14.5$ & 1.5 & $0.4-5.2$ & \\
\hline \multirow[t]{3}{*}{ Auto avaliação } & excelente/m.boa & 43.7 & $34.5-53.0$ & 36.4 & $23.5-51.6$ & 41.8 & $34.3-49.7$ & 0,638 \\
\hline & boa & 38.3 & $29.8-47.6$ & 40.3 & $26.8-55.3$ & 38.8 & $31.5-46.7$ & \\
\hline & regular/ruim & 17.9 & $11.9-26.1$ & 23.3 & $13.1-38.0$ & 19.4 & $13.9-26.3$ & \\
\hline
\end{tabular}

No entanto, as razões de prevalência, quando estimadas por meio do modelo múltiplo de Poisson, indicam que os fatores que influem no perfil de acesso às atividades da USF são afiliação ao subsistema privado e renda. A variável trabalho deixa de ser significativa, quando controlada por idade, sexo e demais variáveis (Tabela 2).

Parte-se agora para a descrição do perfil de acesso aos serviços ofertados pela e na USF, com os resultados tanto para o conjunto da população cadastrada quanto para o grupo parcial, considerando os últimos 12 meses (Tabela 3). Chama atenção que os serviços de médico da família, consulta de enfermagem, dispensação de medicamentos, acolhimento, vacinação não ultrapassem isoladamente $18,6 \%$ da população cadastrada; evidentemente os percentuais são bastante superiores quando se observa apenas o grupo parcial com $100 \%$ de consulta no médico da fa- mília, 51,8\% na dispensação de medicamentos, porém as consultas de enfermagem atingem apenas $11,1 \%$.

Para apenas $11 \%$ da população cadastrada a USF foi o único serviço de saúde utilizado no último ano; $69,9 \%$ utilizou outro serviço de saúde no mesmo período. Cabe ressaltar, que a procura a outro serviço, não foi resultado de eficiente sistema de referencia e contrarreferência, visto que apenas 4 pacientes foram encaminhados pela USF e a maioria dos atendimentos ocorreu em horário comercial.

Detendo-se na visita do ACS, a maioria dos entrevistados considerou a duração da visita adequada, sendo que para 52,0\% este encontro teve duração inferior a 20 minutos. No geral, o ACS responde a todas as perguntas, entretanto $22,2 \%$ dos entrevistados não fizeram nenhuma pergunta, todos os usuários referiram ser tratados com 
Tabela 2. Prevalência do uso residual do PSF e razões de prevalência (RP) nos modelos de regressão simples e múltiplo, segundo variáveis demográficas, socioeconômicas e de saúde.

\begin{tabular}{|c|c|c|c|c|c|c|c|}
\hline \multirow[b]{2}{*}{ Variável } & \multirow[b]{2}{*}{ Categoria } & \multirow[b]{2}{*}{$\begin{array}{c}\text { Prev. } \\
\text { p }\end{array}$} & \multicolumn{2}{|c|}{ Modelo simples } & \multicolumn{2}{|c|}{ Modelo múltiplo } & \multirow{2}{*}{$\begin{array}{l}\text { Valor } \\
\text { de } \mathbf{p}^{*}\end{array}$} \\
\hline & & & $\mathbf{R P}$ & $\mathrm{IC}_{95 \%}$ & $\mathbf{R P}$ & $\mathrm{IC}_{95 \%}$ & \\
\hline \multirow[t]{2}{*}{ Sexo } & feminino & 67,6 & 1 & - & 1 & - & - \\
\hline & masculino & 80,3 & 1,19 & $0.99-1.43$ & 1,16 & $1,00-1,36$ & 0,053 \\
\hline \multirow[t]{4}{*}{ Idade } & $0-11$ & 61,6 & 1 & & 1 & - & - \\
\hline & $20-39$ & 80,5 & 1,31 & $0,94-1,81$ & 1,18 & $0,93-1,50$ & 0,159 \\
\hline & $40-59$ & 86,1 & 1,40 & $1,03-1,91$ & 1,28 & $1,01-1,61$ & 0,041 \\
\hline & $60 \mathrm{e}+$ & 56,2 & 0,91 & $0,60-1,39$ & 0,86 & $0,60-1,24$ & 0,416 \\
\hline \multirow[t]{4}{*}{ Situação conjugal } & casado & 71,6 & 1 & - & - & - & - \\
\hline & separado & 83,3 & 1.16 & $0.87-1.56$ & - & - & - \\
\hline & solteiro & 74,5 & 1.04 & $0.85-1.27$ & - & - & - \\
\hline & viúvo & 70,4 & 0.98 & $0.63-1.52$ & - & - & - \\
\hline \multirow[t]{2}{*}{ Cor autorreferida } & branco & 75,4 & 1 & - & - & - & - \\
\hline & pardo ou negro & 62,5 & 0,83 & $0.61-1.13$ & - & - & - \\
\hline \multirow[t]{2}{*}{ Trabalho } & não & 62,1 & 1 & - & - & - & - \\
\hline & $\operatorname{sim}$ & 84,2 & & & - & - & - \\
\hline \multirow[t]{2}{*}{ Setor supletivo } & não & 45,7 & 1 & - & 1 & - & - \\
\hline & $\operatorname{sim}$ & 85,2 & 1,86 & $1,35-2,57$ & 1,53 & $1,14-2,06$ & 0,005 \\
\hline \multirow[t]{5}{*}{ Renda } & menor $3 \mathrm{SM}$ & 38,8 & 1 & - & 1 & - & - \\
\hline & 3 a $5 \mathrm{SM}$ & 68,3 & 1,76 & $1,08-2,86$ & 1,38 & $0,88-2,17$ & 0,155 \\
\hline & 5 a $10 \mathrm{SM}$ & 85,0 & 2,16 & $1,40-3,42$ & 1,61 & $1,06-2,45$ & 0,025 \\
\hline & $10 \mathrm{ou}+\mathrm{SM}$ & 96,0 & 2,47 & $1,60-3,83$ & 1,64 & $1,07-2,49$ & 0,022 \\
\hline & não informada & 94,5 & 2,44 & $1,56-3,81$ & 1,83 & $1,19-2,81$ & 0,006 \\
\hline \multirow[t]{4}{*}{ Escolaridade } & 0 a 7 & 61,2 & 1 & - & - & - & - \\
\hline & 8 a 10 & 73,3 & 1,20 & $0,86-1,66$ & - & - & - \\
\hline & 11 ou mais & 82,1 & 1,34 & $1,05-1,70$ & - & - & - \\
\hline & não informado & 44,2 & 0,72 & $0,17-3,06$ & - & - & - \\
\hline \multirow[t]{3}{*}{ Auto avaliação } & excelente/m.boa & 77,0 & 1 & - & - & - & - \\
\hline & boa & 72,7 & 0,94 & $0,77-1,15$ & - & - & - \\
\hline & regular/ruim & 68,3 & 0,89 & $0,67-0,16$ & - & - & - \\
\hline
\end{tabular}

* teste t na regressão múltipla

Tabela 3. Percentual de utilização de serviços ofertados pela USF no último ano pela população cadastrada total e grupo de uso parcial.

\begin{tabular}{lcc}
\hline Serviço utilizado & $\begin{array}{c}\text { Grupo uso } \\
\text { parcial }\end{array}$ & $\begin{array}{c}\text { População } \\
\text { cadastrada }\end{array}$ \\
\hline Médico de família & 100,0 & 18,6 \\
Enfermeira & 11,0 & 4,1 \\
Farmácia & 51,8 & 13,6 \\
Acolhimento & 31,4 & 6,5 \\
Vacina & 18,5 & 6,0 \\
Aferição de PA & 53,7 & 17,3 \\
\hline
\end{tabular}

cortesia e respeito pelo ACS. As principais atividades do ACS na moradia foram: questionamentos sobre a saúde dos moradores $(44,3 \%)$, orien- tações de uma forma geral $(27,2 \%)$, conversas informais $(21,5 \%)$ e marcação de exames e consultas $(15,3 \%)$.

Dentre os entrevistados que marcaram consulta de rotina com o médico de família a maioria conseguiu obtê-la em intervalo inferior a 30 dias, observou-se predominância do intervalo entre 20 a 29 minutos na duração referida da última consulta médica. Independentemente da duração o tempo despendido pelo médico foi considerado adequado por todos os entrevistados. O duração da espera entre a chegada na USF e o início da consulta foi de menos de 30 minutos para $36,7 \%$ dos entrevistados e entre 31 e $59 \mathrm{mi}$ nutos para $25 \%$ destes. Todos referiram que o médico respondeu às suas perguntas, sendo que apenas 3,3\% dos entrevistados do não se sentiram à vontade para fazê-las. Em $60 \%$ das consultas realizadas foram solicitados exames. 


\section{Discussão}

A metodologia empregada permitiu captar distintos padrões de utilização de serviços de saúde pela população adstrita ao PSF, os quais estariam encobertos em abordagens que consideram homogênea esta utilização. Apesar da imensa potencialidade das propostas inovadoras incorporadas pelo PSF, observa-se no presente estudo que há um uso seletivo das atividades do PSF pela população cadastrada, fortemente influenciado pela afiliação ao subsistema privado e pela renda. A exceção reside na visita do ACS, atividade obrigatória para todas as famílias cadastradas. Consideramos que conhecer estes perfis do acesso realizado contribui para uma melhor organização do sistema de saúde.

O perfil sociodemografico da população que frequenta a USF local é bastante similar ao encontrado por Fernandes et al. ${ }^{13}$ em estudo transversal de base populacional com residentes em áreas de abrangência da Estratégia de Saúde da Família, em Porto Alegre. Na presente investigação observouse que os moradores cadastrados que não frequentam a USF estão na faixa etária entre 21 e 59 anos, trabalham e são afiliados ao subsistema privado da saúde e possuem maior renda. Estas características sugerem que esta parcela da população tem uma inserção mais geral no espaço urbano o que leva a constrangimentos na utilização de equipamentos de saúde que foram configurados numa lógica territorial alicerçada no local de moradia; ademais os horários de funcionamento bastante restritos das unidades não são compatíveis com a maioria das jornadas de trabalho.

O modo como a população compõe sua cesta de serviços a partir do conjunto dos ofertados é um tema que merece ser aprofundado em estudos posteriores. Os dados obtidos indicam uma priorização da consulta médica, dispensação de medicamentos e vacinação, atividades que compõe o rol tradicional da APS, a exceção é o acolhimento, mas como este é quase sempre "obrigatório", pode ser excluído desta lista. A adesão a atividades de promoção e prevenção parece ser mais difícil, seja pelo não reconhecimento das legitimidades destas ou ainda, pelo "padrão ouro" da assistência à saúde culturalmente difundido no país que se edifica na imagem do consultório medico privado do especialista. Na Espanha, observou-se cenário semelhante na análise dos dados do inquérito do sistema espanhol de saúde em 2006 com marcada diferença de acesso ao generalista ou ao especialista dependendo da classificação social do indivíduo, sendo que aqueles com seguro privado tendem a usar mais o especialista do que o generalista ${ }^{27}$.

A visita obrigatória do ACS acaba se configurando como o único contato com a proposta do PSF. Embora as avaliações das visitas sejam positivas na ótica dos entrevistados, não se pode afirmar que o alto percentual referido de conversa informal e a incapacidade dos entrevistados informarem as orientações recebidas sejam bons exemplos de uma visita domiciliar adequada aos propósitos da estratégia da saúde da família.

Quando comparamos os dados da avaliação da consulta médica com os do Survey do Sistema Nacional de Saúde Britânico ${ }^{33}$ observamos percentuais similares na solicitação de exames (60\% no presente estudo e $61 \%$ no britânico em 2009). É interessante que a avaliação da consulta medica no que tange ao tempo da consulta e capacidade do médico em responder as perguntas é próxima de $100 \%$ no nosso caso e de $77 \%$ no Reino Unido em 2011. Isto pode sugerir que avaliar o médico ainda é um procedimento delicado para a população entrevistada. Afinal, a expressão de satisfação do paciente com seu médico recebe influencias da imagem e do papel social dos médicos em cada sociedade ${ }^{34}$.

No título do artigo foi proposto a identificação do acesso realizado às atividades do PSF em área com alta afiliação ao subsistema privado. $\mathrm{Na}$ nossa amostra o percentual de afiliados foi de 70,7\%. Utilizando, como uma aproximação, os dados disponíveis do Sistema de Informação da Atenção Básica (SIAB) ${ }^{35}$ observa-se que no Brasil, em dezembro de 2010, o percentual da população afiliada ao subsistema privado varia de 2,6\% na região Norte até $12,8 \%$ no Sudeste. No estado de São Paulo o percentual é de 16,5\% e no município de Santo André é de 27\%. Deste modo, podemos considerar que o local escolhido foi bastante apropriado para cumprir os objetivos propostos e que os resultados aqui apresentados podem contribuir para um aprofundamento das possibilidades e dos constrangimentos da implantação de unidades de saúde da família em boa parte do território brasileiro. 


\section{Colaboradores}

A Bousquat foi responsável pelo desenho metodológico, análise dos dados e elaboração do manuscrito. MCGP Alves foi responsável pelo desenho da amostra, análise dos dados e elaboração do manuscrito. A Gomes foi responsável pelo trabalho de campo e elaboração do manuscrito.

\section{Agradecimentos}

Ao CNPq/ DECIT pelo financiamento da pesquisa e à Secretaria Municipal de Saúde de Santo André que apoiou o trabalho da nossa equipe.

\section{Referências}

1. Escorel S Giovanella L, Mendonça MHM, Senna MCM. O Programa de Saúde da Família e a construção de um novo modelo para a atenção básica no Brasil. Rev Panam Salud Publica 2007; 21(2):164-176.

2. Marques RM, Mendes Á. Atenção Básica e Programa de Saúde da Família (PSF): novos rumos para a política de saúde e seu financiamento? Cien Saude Colet 2003; 8(2):403-415.

3. Facchini LA, Piccini RX, Tomasi E, Thumé E, Silveira DS, Siqueira FV, Rodrigues MA. Desempenho do PSF no Sul e no Nordeste do Brasil: avaliação institucional e epidemiológica da Atenção Básica. Cien Saude Colet 2006; 11(3):669-681.

4. Viana ALD, Dal Poz MR. A reforma do sistema de saúde no Brasil e o Programa de Saúde da Família. Physis 2005; 15(Supl.):225-264.

5. Sisson MC. Considerações sobre o Programa de Saúde da Família e a promoção de maior equidade na política de saúde. Saude Soc 2007; 16(3):85-91.

6. Nedel FB, Facchini LA, Martín-Mateo M, Vieira LA, Thumé E. Family Health Program and ambulatory care-sensitive conditions in Southern Brazil. Rev Saude Publica 2008; 42(6):1041-1052.

7. Macinko J, Guanais FC, Souza MF. Evaluation of the impact of the Family Health Program on infant mortality in Brazil, 1990-2002. J Epidemiol Community Health 2006; 60(1):13-19.

8. Bousquat A, Cohn A, Elias P E. O PSF e a dinâmica urbana das grandes cidades. In: Viana ALD, Elias PEM, Ibañez N, organizadores. Proteção Social: dilemas e desafios. São Paulo: Hucitec; 2005.

9. Caetano R, Dain S. “O Programa de Saúde da Família e a reestruturação da atenção básica à saúde nos grandes centros urbanos: velhos problemas, novos desafios". Physis Revista de Saúde Coletiva 2002; 12(1): 11-23.

10. Sousa MF, Hamann EM. Programa Saúde da Família no Brasil: uma agenda incompleta? Cien Saude Colet 2009; 14(Supl. 1):1325-1335.

11. Campos FE, Cherchiglia ML, Aguiar RAT. Reflexões sobre Saúde da Família no Brasil: desafios e oportunidades. Revista Brasileira de Saúde da Família 2002; 2(5):71-79.

12. Andersen RM. Revisiting the behavioral model and access to medical care: does it matter? J Health Soc Behav 1995; 36(1):1-10.

13. Fernandes LCL, Bertoldi AD, Barros AJD. Utilização dos serviços de saúde pela população coberta pela Estratégia de Saúde da Família. Rev Saude Publica 2009; 43(4):595-603. 
14. Bousquat A, Alves MCGP, Elias PE. Utilização do Programa de Saúde da Família em regiões metropolitanas: abordagem metodológica. Rev Saude Publica 2008; 42(5):903-906.

15. Santos IS, Ugá, MAD, Porto SM. O mix públicoprivado no Sistema de Saúde Brasileiro: financiamento, oferta e utilização de serviços de saúde. Cien Saude Colet 2008; 13(5):1431-1440.

16. Newacheck PW, Stoddard JJ Hughes DC, Pearl M. Health insurance and access to primary care for children. N Engl J Med. 1998; 338(8):513-519.

17. Harmom C, Nolan B. Health insurance and health services utilization in Ireland. Health Econ 2001; 10(2):135-145.

18. Hullegie P, Klein TJ. The effect of private health insurance on medical care utilization and self-assessed health in Germany. Health Econ 2010; 19(9): 1048-1062.

19. González Alvarez ML, Barranquero AC. Inequalities in health care utilization in Spain due to double insurance coverage: An Oaxaca-Ransom decomposition. Soc Sci Med 2009; 69(5):793-801.

20. Borrell C, Fernández E, Schiaffino A, Benach J, Rajmil L, Villalbí JR, Segura A. Social class inequalities in the use of and access to health services in Catalonia, Spain: what is the influence of supplemental private health insurance? Int J Qual Health Care 2001; 13(2):117-125.

21. Garrido-Cumbrera M, Borrell C, Palència L, Espelt A, Rodríguez-Sanz M, Pasarín MI, Kunst A . Social class inequalities in the utilization of health care and preventive services in Spain, a country with a national health system. Int J Health Serv 2010; 40(3):525-542.

22. Gonzáleza B, Urbanos RM, Ortega P. Oferta pública y privada de servicios sanitarios por comunidades autônomas. Gac Sanit 2004;18(Supl. 1):82-89.

23. Fusté, J, Séculi E, Brugulat $\mathrm{P}$, Medina $\mathrm{A}$, Juncà $\mathrm{S}$. Población con cobertura pública o doble cobertura de aseguramiento sanitario. ¿Cuál es la diferencia? Gac Sanit. 2005; 19(1):15-21.

24. Santos IS. Evidência sobre o mix público-privado em países com cobertura duplicada: agravamento das iniquidades e da segmentação em sistemas nacionais de saúde. Cienc Saude Colet 2011; 16(6): 2743 2752.

25. Sawyer DO, Leite IC, Alexandrino R. Perfis de utilização de serviços de saúde no Brasil. Cienc Saude Colet 2002; 7(4):757-776.
26. Travassos C, Viacava F, Pinheiro RS, Brito AS. Utilização dos serviços de saúde no Brasil: gênero, características familiares e condição social. Rev Panam Salud Publica. 2002; 11(5-6):365-373.

27. Ribeiro MCSA, Barata RB, Almeida MF, Silva ZP. Perfil sociodemográfico e padrão de utilização de serviços de saúde para usuários e não-usuários do SUS - PNAD 2003. Cienc Saude Colet 2006; 11(4): 1011-1022.

28. Basto G, Duca GF, Hallal PC, Santos IS. Utilization of medical services in the public health system in the Southern Brazil. Rev Saude Publica 2011; 45(3): 454-475.

29. Porto SM, Santos IS, Ugá MAD. A utilização de serviços de saúde por sistema de financiamento. Cien Saude Colet 2006; 11(4):895-910.

30. Blay SL, Fillenbaum GG, Andreoli SB, Gastal FL. Equity of access to outpatient care and hospitalization among older community residents in Brazil. Med Care 2008; 46(9):930-937.

31. Instituto Brasileiro de Geografia Estatística (IBGE). [site na Internet]. [acessado 2012 mar 12]. Disponível em: http://www.ibge.gov.br

32. Barros AJD, Hirakata VN. Alternatives for logistic regression in cross-sectional studies: an empirical comparison of models that directly estimate the prevalence ratio. BMC Med Res Methodol. 2003; 3:21.

33. Quare Quality Commission. Tabels Outpatient Survey. [site na Internet]. [acessado 2012 mar 12]. Disponível em: http://www.cqc.org.uk/public/reportssurveys-and-reviews/surveys/outpatient-survey-2011

34. Reimann S, Strech D. The representantion of patient experience and satisfaction in physician rating sites. A criteria-based analysis of English and Germany sites. BMC Health Serv Res 2010; 10:332.

35. Brasil. Ministério da Saúde (MS). [site na Internet]. [acessado 2012 mar 12]. Disponível em: http://www. datasus.gov.br

Artigo apresentado em 30/04/2012

Aprovado em 17/07/1012

Versão final apresentada em 30/08/2012 